This item was submitted to Loughborough's Research Repository by the author.

Items in Figshare are protected by copyright, with all rights reserved, unless otherwise indicated.

\title{
Design methodology for integrating multipath systems (building services)
}

PLEASE CITE THE PUBLISHED VERSION

http://dx.doi.org/10.1108/JEDT-09-2011-0057

\section{PUBLISHER}

(C) Emerald

\section{VERSION}

AM (Accepted Manuscript)

\section{PUBLISHER STATEMENT}

This work is made available according to the conditions of the Creative Commons Attribution-NonCommercialNoDerivatives 4.0 International (CC BY-NC-ND 4.0) licence. Full details of this licence are available at: https://creativecommons.org/licenses/by-nc-nd/4.0/

\section{LICENCE}

CC BY-NC-ND 4.0

\section{REPOSITORY RECORD}

Fouchal, Farid, Tarek M. Hassan, and Dennis L. Loveday. 2019. "Design Methodology for Integrating Multipath Systems (building Services)". figshare. https://hdl.handle.net/2134/23154. 


\title{
Design Methodology for Integrating Multipath Systems (Building Services)
}

Fouchal,F ,Hassan,TM ,Loveday,DL,

School of Civil and Building Engineering, Loughborough University, UK

\begin{abstract}
Purpose: Most multipath delivery systems, such as Building-Services (BSs) are arbitrary distributed with no known solution to reduce the complexity in the way channels are arranged. This paper reports on a geometrical integration methodology that can be used to organise some types of these systems.
\end{abstract}

Methodology: Integration for optimal functionality through reduction of geometrical complexity is achieved by: (i) understanding the elements of complexity within current practices; (ii) identifying commonalities between the various components which can be used for integration; (iii) performing an axiomatic design to resolve design complications; (iv) adopting theory of inventive problem solving for methodology and process development toward optimal functionality (v) generating a mathematical solution to inform digital modelling of optimal design.

The study takes into account thermophysical and electromagnetic interactions between utilities; and uses novel mathematical manipulations based on designing a manifold of spherical and cylindrical geometries joined using Bezier surfaces.

Findings: Once a solution was reached Computer Aided Design iterations were undertaken for channelling six BSs into a single unit. The outcome was concentric cylindrical-spherical shells superimposed with spacings of typically few millimetres to deliver/distribute the utilities. Applied to bring together BSs into a single trunking system at minimal, yet appropriate, proximal distances and allows distribution of any number of services in any direction. Physical prototypes were produced and initial testing of their performance (reported elsewhere) has been encouraging.

\section{Originality:}


A design methodology for integrating arbitrary distributed paths/conduits. The approach could be incorporated into CAD tools as a design feature to facilitate integration of multipath delivery systems.

Keywords: Building Technology, Computer Aided Manufacturing and Design, Advanced Assembly Methods, Modelling, Rapid Prototyping, Manufacturing Engineering.

\section{Introduction}

The core of this research work is a design feature for integrating some types of arbitrary laid down curves or distributed conduits. The approach being described has emerged while searching for a solution to reduce the complexity encountered in attempting to physically integrate Building Services (BSs) systems into a single conduit. The BSs case study represents a more generic problem which is encountered in trials for geometrically or physically 'bundling' or integrating curves in 3D space (i.e. of utilities/services). A number of previous approaches (theoretical) have been proved to simplify these complexities; among these is the axiomatic design (Nam, 2001; Nam, 1990), which resolves design issues via suboptimal compromises providing a systematic analysis for transformation (progression) to a standardised functional requirement. At the same time, the theory of inventive problem solving (Terninko, 1998; Domb, 1998) guides methodologies and process development toward optimal functionality. In the current work, we aim at optimal functionality through design solution by reduction of geometrical complexity (encountered for example in utility pathways as in buildings).

The chosen case study in this research work was the installations of multiple building services systems into buildings. Such systems are becoming increasingly technologically complex, for which their design and installation requires the involvement of numerous professions and trades. Designers and contractors working on the same project are frequently employed by different companies. The coordination of these participants and subsystems is crucially important for the efficiency of the installation process and the performance of the system as a whole. The involvement of several trades over a series of 'Fixes' increases the risk of practical problems being encountered, as well as increased time and cost of construction. This is directly linked to the geometrical complexity as they are separately distributed in an arbitrary manner throughout the building. If it was possible to integrate the installation of these utilities into a single fix by a single trade, then it would offer many advantages. Integration is crucial and its importance is widely recognised already in other sectors such as aerospace and defence sectors where it has led to a major shift in procurement procedures. 
The primary objective of the novel methodology being proposed and developed here is thus to bring together all the BSs to within minimal, yet appropriate, proximal distances and into a single optimal trunking network for distribution around a building. This approach has been explored through novel mathematical manipulations, Computer Aided Design (CAD) modelling and implementation of Additive Layer Manufacturing (ALM) capability as a production method. Fabrication of fully optimised 3D CAD models of physically integrated and embedded utilities is challenging as it required incorporating and fabricating channels and ducting simultaneously to produce whole single working elements within a wall section or in confined spaces. ALM is a direct production of finished goods using additive processing from digital data, which eliminates all tooling and with design possibilities that are virtually limitless - rather than Design for Manufacture. One of the principal advantages of ALM is the ability to manufacture working assemblies and linkages straight off the machine. The resulting methodology can enable design and production of a multi service trunking system (MSTS) that is based on nodes (spherical) and runs (elongated cylinders). A node is the position along the utility pipe, cable or/and duct at which distribution in more than one direction is required by splitting into two or more pipes, cables or ducts. The proposed concept is developed by taking into consideration current building regulations/standards for the following services: hot and cold water, ventilation (hot and cold), electricity supply and data cabling.

New mathematical development has permitted conversion of the conventional utility delivery conduits (pipes, duct, etc) which are arbitrarily distributed in 3D space to an integrated and parameterised system of channels which is based on concentric 3D surfaces positioned in a novel fashion for routing the services through a building, whilst preserving required spacing between services. This approach is detailed in this paper.

It is a designed shell type arrangement of the channels which are concentric and superposed on each other (at few millimetres spacings), combining spherical and cylindrical geometries. This setup displays all the channels in a way that allows access to any inter-layer space (space between two consecutive surfaces which is in fact the channel) from any orientation penetrating through any other layers. The proximal rule of separation between services is respected by keeping empty gaps around the penetrating channel and the corresponding layers being crossed. On the "node" any number, n, of deliveries and distribution of services in any, m, directions could be made. On the "runs", services are delivered using a geometrically matched set of concentric cylinders made to connect in a simple manner to the spherical nodes. 


\section{State-of-the-art}

A literature review was conducted to reveal whether any design procedure was available to geometrically integrate any arbitrarily-distributed pattern of multi path delivery systems; and to discover whether BSs had been previously integrated in any manner prior to this work.

Axiomatic Design (AD) was explored first, as it provides a rational and a scientific way to develop and assess design alternatives, AD also pre-emptively addresses and resolves design issues that traditionally are discovered so late that only suboptimal compromises are possible (Hamilton, 2006). AD is a system design methodology which uses matrix methods to systematically analyze the transformation of any kind of needs into functional requirements, design parameters, and process variables (Nam, 2001; Nam, 1990). Furthermore, in addition to the Taguchi approach (Anderson, 2000) and to the robust design_ Quality Function Deployment which emerged in the West in the early 1980s, some are claiming that the theory of incentive problem solving (TRIZ) completes the trio (Terninko, 1998). Whether this is the case, time alone will tell, but clearly industrial interest is growing (Domb, 1998). When comparing AD to TRIZ to understand how one can come up with a consistent approach for the geometrical complexity problem being studied, two main topics were studied for the purpose of this research work, as follows: (Mann, 1999) Corollary 2 - Minimisation of functional requirements (FRs) and constraints. This corollary states that as the number of functional requirements and constraints increases, the system becomes more complex and thus the information content is increased. This corollary recommends that the designer strive for maximum simplicity in overall design or utmost simplicity in physical and functional characteristics. In parallel, TRIZ ideal final result (IFR) philosophy (which corresponds to corollary 2 in AD) states that a system is a "fee" for realisation of the required function and IFR will be realised if the system does not exist but the required function is performed. IFR helps an engineer focus on concepts that minimise requirements in substance, energy, and complexity of engineering product and process. Corollary 3 in $\mathrm{AD}$ - which is about integration of physical parts, integrates design features into a single physical process, device or system when physical requirements (PRs) can be independently satisfied in the proposed solution. This corollary states that the number of physical components should be reduced through integration of parts without coupling functional requirements. However, more physical integration is not desirable if it results in an increase of information content or in a coupling of FRs. Evolution pattern 5 from TRIZ, which is about increased complexity followed by simplification states that the technological systems tend to develop first toward increased complexity (i.e., increased quantity and quality of system functions) and then toward simplification (where the same or better performance is provided by less complex system). Here we recall the current status of BSs distribution and the need for an integration solution to relief the complexity and its associated problems. The term mo-bi-poly indicates that mono-function products evolve into bi-function or poly-function products through integration of physical embodiments. 
Setting the scene for consideration of the integration solution being developed in this research as a design feature into CAD, through adoption of the logic used by Noort et al. (2002), who defined form features as regions of the part that have some functional meaning. The form features contain class-specific design information that is captured by means of feature elements and feature constraints. Feature elements are shapes and userdefined variables. Features constraints can be, for example, a geometric distance faceface constraint, a dimension constraint, which specifies a dimension to be within a given range, an on-boundary constraint, which specifies feature face to be on the boundary of the part. The data structure of cylindrical features consists of circular edges and cylindrical face. The circular edge is a set of connected edges that may form the closed boundary of a non-self-intersecting face. Non-linear (curve/circular) edges are formed by cylindrical or conical faces.

Another review performed to discover whether BSs were integrated in any manner was application oriented. In an investigation on the problems within the BSs industries conducted by John (2002), methods and solutions to overcome these problems were proposed and the Integrated Logistic Support (ILS) techniques and their applications were introduced and described for BSs in the construction sector. One of the major benefits of this approach is that the operational and maintenance cost of the BSs systems would be identified earlier on in the project and optimised for minimum through life-cost. The whole-building integration principle, similar to the process used in the production of automobiles which is designed and manufactured to optimise car performance, can be extended to include more physical integration of the different parts of the building. At the moment, whole-building integration works the same way as for the automotive industry, only it achieves high-performance buildings, which begins with computer-simulated design to analysis of building components and systems and then it integrates them so that the overall building performance is optimised. The national building survey by Amory Lovins (Lovins, 1992; Lovins, 1991) concluded that: "A lack of integration in the design process has made buildings costlier to build, costlier to cool, and less comfortable than they should be." This situation remains true today, especially for HVAC systems that are being added to buildings rather than being integrated into them. In fact, no matter how industrialised the construction sector is trying to be each building is at the end bespoke rather than mass-produced product. The current drive within the sector is towards leaner, better Modern Methods of Construction (Lovell, 2003), which undoubtedly can be reinforced by serious uptake of physical integration at every level of the construction processes from design all the way through construction to usage. Bemtgen (2006) proposed a detailed approach to make a transition from conceptual integration to physical integration. This starts with innovative planning and architecture, and then is followed by choosing Low-Energy materials, innovative components, and taking advantage of the innovation in Building Management Systems (BMS) including monitoring to finally succeed in physical integration. 
There have been various views on how integration of building structural and functional components could improve the construction cost, the running costs and improve the user comfort (Viadya, 2009). However, the integrations reported-on in these views were limited to the control and the operational aspects, with very little development being carried out on physically integrating the parts and the components that form buildings in general. As suggested by this review, there is a lack of knowledge on how to reduce the complexity encountered in physically integrating building services system into a single unit.

The current investigation was conducted in light of a wider scope which was a search for a methodology for geometrically or physically bundling or integrating of any number of curves in 3D space. The aim of the research is consequently to develop a design feature for intelligently-integrating many types of randomly laid curves, distributed conduits or multi path delivery system such as distribution of services in a building. We have therefore engaged into the development of a solution for integrating BSs based on a mathematical development which dictated the geometrical solution implemented using CAD surface modelling tools. The generated 3D models were then partly produced by ALM technology and partly by conventional manufacturing methods aiming for a sustainable solution, reduction in energy wastage during manufacturing, gain in material used for production and reduction in labour cost at various levels (handling, installation and maintenance) as described in this paper. This work addresses directly the challenges of physical integration of building services into a single trunking system and demonstrates through the proposed multi services trunking system (MSTS) solution a more generic methodology. It shows how one can possibly develop, integrate any complex systems and provide a solution starting from fundamental understanding of the problems causing the complexity down to using CAD and ALM to produce a demonstrator prototype of the solution.

\section{Methodology}

To come up with a methodology to physically integrate any type of multi-path distribution or delivery system, an emphasis was placed on the integration of BSs as a strong representative case of the more generic problem. The expected difficulties to be overcome were related to the design practices of the architects, the designers, and the service engineers, and the current manufacturing methods applied in construction, as well as issues related to materials performance together with safety and longevity.

\subsection{Standards and regulations}


The list of multi-path distribution or delivery systems is very long, among which are pipe networks for transporting compressible, incompressible, gas-liquid two phase, settling slurries or non-Newtonian fluids (such as for BSs and for chemical plants, pharmaceutical and medical, or for automotive and aviation, or for naval construction). In the current study, the preliminary design integration was based on the case of BSs and was restricted to incorporate the following: hot and cold water for which a new connection protocol will be required as potable or for central heating; air for ventilation and/or for air conditioning; data; and electric mains for which a new dedicated physical interface will be required. All designs and calculations were undertaken for a case study which comprised a hypothetical open plan office designed for twenty people. The following adopted parameters were based on the currently-applied standard regulations, of the United Kingdom. These include:

- Water Supply (Water Fittings) Regulations and their Schedules which are Statutory Instruments (1999 No. 1148 and No. 1506) (see Water Supply 1999);

- The British Standards: BS6700 (for services supplying water), BS CR 1752 and BS EN 1505 (for ventilation for buildings), BS 7671 and BS6231 (requirements for electrical installations);

- Physical laws that included Bernoulli's theorem for flow-metering; D'Arcy fluid flow and Fourier's Law of heat conduction (Liu 1990, Liu 1972);

- Potable water proximities were calculated under the standard BS6700, where we have found that a $21 \mathrm{~mm}$ ID pipe will satisfy the water requirements for the chosen case study;

- Calculations for ventilation under standard BS CR 1752 and BS EN 1505 suggested that a prescribed flow rate of $8 \mathrm{~L} / \mathrm{s} /$ occupant of fresh air which summed for 20 people can be carried by a duct of 436-483 mm ID when conventional flow rates are applied;

- For electric power under the standard BS 7671 and BS6231, $15 \mathrm{~kW}, 240$ volts single phase AC @ 60 Amps is needed to satisfy all personnel and equipment requirements of the open plan office;

- For data the category 8 (CAT8) cables which are a 4 x 22/1 AWG will be adequate and will satisfy the needs for more digital applications in the future.

Determination of proximal distances was based on achieving minimal energy loss or minimum heat exchange between utilities and ensuring zero leakage especially of water toward electricity cables as well as current regulations that govern services installation. These measures have to account for thermal insulation for which a geometrical positioning of all these services is needed to maintain compliance with current building 
regulations. The following sections will address the essential elements of this integration challenge, while the basic integration criteria are: (1) respecting the proximal rules for safety, (2) respecting the proximal rules for optimal thermal insulation between hot and cold utilities, (3) performing distribution without breaching the proximal rule and (4) allowing for an efficient joining and connectivity of the different components.

\subsection{Mathematical development}

The methodology undertaken to develop a multi path integrated system for BSs is based on the findings from a review of the current building standards, the findings from a physics study that was conducted to understand the interactions (thermal, electromagnetic) between utilities, together with a mathematical development to allow integration of BSs into a single trunking permitting distribution in all directions. The mathematical developments consist of geometrical arrangements for designing a manifold by coupling spherical and cylindrical geometries using Bezier surfaces (Wilson, 2005). This was conducted in an attempt to solve the problem of transition from an "impossible" distribution (i.e. the proximity rule cannot be satisfied) of services if needed in more than two directions by simply bundling them in 3D curved fashion, to a new design which would allow flexible distribution in any number of directions. This solution is also intended for $r$ integration of BS utilities of the future into building construction walls, panels or for placement in a confined space within a building as a first step toward potentially next generation BSs. Also in these integrations the required steps included defining the interactions between utilities and the materials being used to make the MSTS. This investigation additionally took into account the current building regulations. The first step was to conduct a physics study to define the permissible distances between the utilities once brought together in a bundle assembly. For the present design approach, the intended utilities to be transported/delivered by the MSTS are considered to be: water (hot and cold), power (12volts, 240 volts), data (5volts signal, optical signal, etc.) and air for ventilation (heated and cooled). Once integration in concept is geometrically reached the 3D CAD models are produced, and evaluation of the solution was carried out against the basic integration criteria. A variety of sensor components were also embedded into the MSTS for communication with the building operating systems on functionality issues as well as for informing on the user's activity. Each stage of the analytical process is described in the sections that follow.

The conventional methods used for distributing services in buildings consist of a set of different routes throughout the building (of pipes, ducts or cables) for each service (such as hot and cold water, ventilation, electricity, data, and return water). These routes extend from the main entry point as the utility arrives at a building all the way to the points where they are needed. A transition from the current conventional way of thinking to a new concept (where all services are integrated) is via a single trunk comprising a set of routes. These routes are related by a mathematical function such that they (for all 
utilities) stay at a fixed distance from a common trajectory (which is calculated in 3D space) while they distribute throughout the building. This concept uses anew geometrical form for the channels (pipes, ducts), which involves reshaping the external surfaces of the conventional tubing/cylinder into a set of concentric pairs of surfaces where the space between each pair makes the channel. They are concentric around a common trajectory which encompasses the linking medium (i.e. integrating them) for all surfaces and corresponding channels. These comprise overlapping 3D concentric layers of spheres ("nodes") which are intermittently joined to long cylinders ("runs"). Within the node, services can be eventually distributed in any number of directions. The overlapping channels are therefore trapped between the concentric surfaces along the straight runs which are linked by corresponding gaps between the concentric spherical surfaces at the nodes. The node is designed to perform services distribution without breaching proximal rules; any given utility is therefore allowed to cross other utilities layers under provision of a partly blocked peripheral area around it within the crossed layer to maintain the proximity rule between services as described in the following section.

The suggested transition required complex mathematical manipulation which will take into consideration usage of minimum space occupation within the building and allow for full geometrical integration of BSs by means of the Multi Services Trunking System (MSTS) concept. This transition is twofold; first, we have to define the types of the 3D surfaces to be used (here they should be boundaries of cylindrical cavities, these are trapped between layers that are concentric and extend along a common axis/trajectory from the point of entrance into the building to the position in the building where the utility is required), and then second, we have to propose a solution to link these surfaces together. The solution identified to potentially answer the question is based on coupled spherical and cylindrical shapes which are already overlapped into manifolds and joined using harmonised Bezier surfaces. The MSTS manifold will contain as many overlapping (superimposed) concentric spaces as needed to deliver an "infinite" number of services and distribute them without breaching the calculated proximity rules as opposed to the initial solutions (given in section 5).

The Bezier surfaces will serve as exact linking surfaces between the spherical and the cylindrical surfaces to form a homogeneous surface such as a manifold. To clarify the cubic Bezier curve method defined by four points, where two are endpoints, (x0, y0) is the origin endpoint, $(\mathrm{x} 3, \mathrm{y} 3)$ is the destination endpoint, the points $(\mathrm{x} 1, \mathrm{y} 1)$ and $(\mathrm{x} 2, \mathrm{y} 2)$ are the control points. Two equations are used to define the points on the curve; both are evaluated for an arbitrary number of values t, which is between 0 and 1 . One equation yields values for $\mathrm{x}$, the other yields values for $\mathrm{y}$, and as increasing values for $\mathrm{t}$, are supplied to the equations, the point defined by $\mathrm{x}(\mathrm{t}), \mathrm{y}(\mathrm{t})$ moves from the origin to the destination. The following shows how the equations are defined:

$$
x(t)=a x t_{3}+b x t_{2}+c x t+x_{0}
$$


where a, b and c are constants.

$$
\begin{gathered}
x_{1}=x_{0}+x c / 3 \\
x_{2}=x_{1}+(c x+b x) / 3 \\
x_{3}=x_{0}+c x+b x+a x \\
y(t)=a y t_{3}+b y t_{2}+c y t+y_{0} \\
y_{1}=y_{0}+c y / 3 \\
y_{2}=y_{1}+(c y+b y) / 3 \\
y_{3}=y_{0}+c y+b y+a y
\end{gathered}
$$

This method can be reverse-engineered to give the coefficient values based on the points described above in the following set of equations:

$$
\begin{aligned}
& c x=3\left(x_{1}-x_{0}\right) \\
& b x=3\left(x_{2}-x_{1}\right)-c x \\
& a x=x_{3}-x_{0}-c x-b x \\
& c y=3\left(y_{1}-y_{0}\right) \\
& b y=3\left(y_{2}-y_{1}\right)-c y \\
& a y=y_{3}-y_{0}-c y-b y
\end{aligned}
$$

Now, simply by knowing coordinates for any four points, we can create the equations for a simple Bezier curve. To create a Bezier surface, we blend a mesh of Bezier curves using the blending function

$$
P(u, v)=\sum_{j=0}^{m} \sum_{k=0}^{n} p_{j, k} B E Z_{j, m}(v) B E Z_{k, n}(u)
$$

where $\mathrm{j}$ and $\mathrm{k}$ are points in parametric space and ${ }^{p_{j, k}}$ represents the location of the knots in real space. The Bezier functions specify the weighting of a particular knot, which are the Bernstein coefficients. The definition of the Bezier functions is given as:

$$
B E Z_{k, n}(u)=C(n, k) u^{k}(1-u)^{\mu-k}
$$

where $C(n, k)$ represents a binary coefficient; when $u=0$, the function is one for $\mathrm{k}=0$ and zero for all other points. When we combine two orthogonal parameters, we find a Bezier curve along each edge of the surface, as defined by the points along that edge. Bezier surfaces are useful for interactive design. In the current situation, it is a matter of defining the entire services requirement at different locations in a building then calculating the 
optimal pathway within the whole building for the integrated building services solution through the MSTS concept. The next step is to calculate the different cross section areas needed for all utilities throughout the building. Once these parameters are known at every point of the calculated pathway the cross section areas will be used to generate the spatial (volumetric) passage way for each service, however the proximity rule will be breached in the first instance at distribution points. To overcome this problem, an extra dimension will be required to solve the problem by passage from 2D channelling type of piping arrangement (as in the initial solution - which can be seen in the following section (3.3)) to 3D staked spaces on a manifold form surfaces. This manifold geometric form will not respect the Poincare conjuncture as it is supposed to possess edges at connection points which do contradict the no boundary rule, it can also be extended to infinity which implies that it is not compacted and also it is not simply connected. As a result, our solution will be a 3D manifold that is not homeomorphic that in many ways is the ideal geometric solution for the MSTS, and allows distribution of services in any direction without breach of the proximity rule.

This discussion leads us to a conclusion that combining spherical and cylindrical shapes to create a base surface for the MSTS and usage of Bezier surfaces as a connecting technique to link them to generate an ideal 3D manifold as the solution for geometrical complexity problem.

\subsection{CAD Modeling}

The functional multi-services trunking (run \& node) designs were carried out in CAD using Rhino software, and can be seen in figure 1. They represent a succession of concentric shells (sphere and cylinder linked by Bezier surfaces) containing an internal series of empty concentric gaps to act as conduits for services or as voids ready to be filled with thermally insulating material such as silicon sealant, or electrically insulting material, or even an electromagnetic shielding material such as low melting alloys, these being an integral part of the solution. The empty gaps are proposed to serve as channels for services in a similar manner to pipes and ducts that are conventionally used for plumbing and ventilation. The node is particularly innovative in that it guarantees any number of distributions of services in all directions, while runs are designed to cross long distances within a building to link the distribution nodes.

One of the arrangements of the utilities requirement as calculated for a 20-person open space office in the case study selected for design purpose in this study is given as cross section in figure 2. 


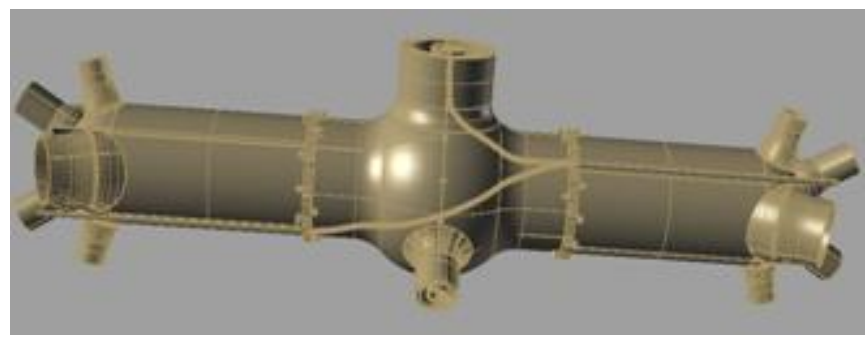

Figure 1 Optimised novel solutions - MSTS

Outer shell/O-ring groove

Inner Low Melting Alloy

Inner water shell

Inner Silicon/outer ventilation

Inner ventilation

Inner silicon

Inner water

Inner Low Melting Alloy

Data twisted cable

Electric cables

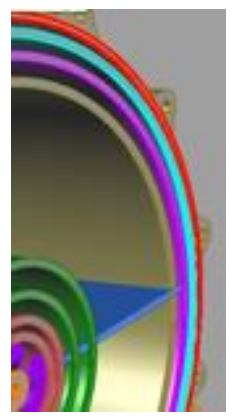


Figure 2 Coloured illustration of an optimised MSTS for 20-person open plan office case study

The final CAD complex geometries shown in figure 1 could be physically produced using a combination of two manufacturing technologies; one could be conventional to produce the runs directly by simple extrusion of a corresponding cross sectional pattern while the other geometrically complex part that is the node (which is shown in figure 3) could be produced by an advanced manufacturing technique such as ALM.

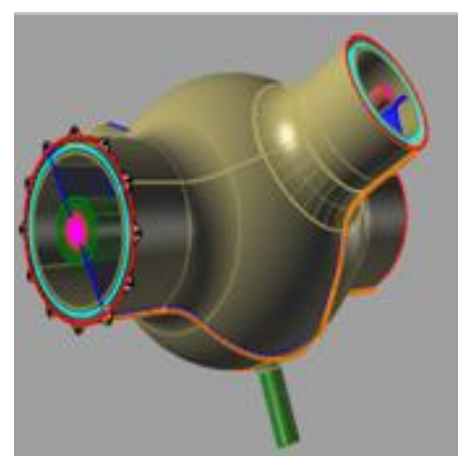

Figure 3 Distribution "Node" for the optimised novel solution - MSTS

When investigated, the physics behind the standards for data cabling has revealed that laying down signal/data cables into a spiral form will attenuate electromagnetic interferences which are prone to disturb the signal within the cables. This geometrical technique is generally reinforced by a metal based protection around the spiral to further enhance the shielding from interferences created by the electric current within a nearby mains electric power ( 240 volts) cable. The spiral shaped channels for data cabling were adopted in our design and incorporated in the CAD models (see figure 4) as a set of eight channels in tubes which are arranged in spirals around the central axis of the MSTS. Around this bundle of spiral channels is an external layer of low melting alloy to act as an electromagnetic shield which is filled in the empty space between the pair of the nearest concentric surfaces all the way along the length of the runs and nodes to produce a true physical replica of a standard CAT8 type data cable. 


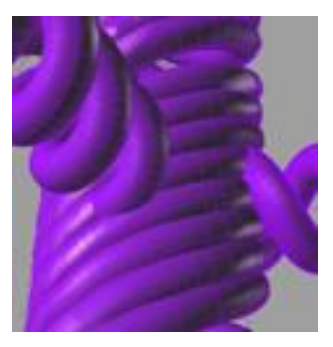

$\underline{\text { Figure } 4}$ Channels for data cable in spiral shape to attenuate interferences

A 3D splitting plan as shown in figure 5 can also be embedded bisectionally in the MSTS to multiply the number of channels by a factor which is equal to the number of splitting plans times two, allowing more utilities to be distributed.

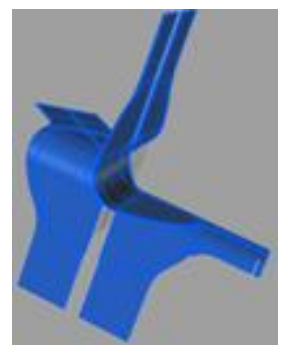

$\underline{\text { Figure } 5}$ Splitting plan dividing the MSTS concentric channels to increase the number of conduits within the trunking

\subsection{Physical MSTS prototype}

Using ALM prototype MSTS will be produced, and its ability to deliver water, air, electricity and data will be investigated in the laboratory. This new concept for services delivery will be compared with the existing standard methods in terms of factors that include integrity and safety of the services delivery, space requirements, installation time, connectivity, energy implications (operational and embodied). The experimental prototypes were also used for the assessment of material requirements in terms of manufacturability and usability. Several existing materials for ALM processes were tested and evaluated by producing prototypes while refining the design and adding 
functionalities. These materials were polymers which were specifically designed for Stereolithography systems. A number of thermal insulation materials for filling in between the MSTS empty voids were also tested as they may improve the overall thermal insulation of the walls and the physical integrity of the trunking. These were high viscosity silicon sealants which were forced into the narrow gaps between the concentric shells of the MSTS. Furthermore, a low melting alloy material was also filled into the space around the spiral channels for data; this was the MCP 47 which has a low melting point of $47 \square \mathrm{C}$. When taking the outcome of the design to the application field, some of the materials tested within the produced prototypes during this research work showed some degrees of limitation which will require more effort, time and cost to address. However, there are already some promising new materials being developed using nanotechnology-engineered compositions (such as ceramic or nano-clay particles filled polymers) to be considered for the MSTS, but this is outside the scope of this paper.

\section{Discussion}

A methodology has been developed and tested to geometrically integrate some types of arbitrarily-distributed multi-path delivery systems. The case study adopted in this paper was that of the building services utilities distributed around a building. The potential advantages offered by embedding all the building services utilities into a single trunking might lead to the reduction in the space they occupy as well as minimising times for installation and numbers of trades required. This can potentially lead to reduction in costs of the buildings of the future, based on a number of targets including singularity of materials, confinement of services to reduce energy wastage and possibly energy recuperation. Besides there-configurability, flexibility and adaptability of the building spaces to suit different user's requirements at different stages of the building life is also a major advantage. A direct reduction in the labour work and an important saving on energy and time of manufacturing, assembly and maintenance might also be achieved together with near zero wastage during the production process which is possible through ALM.

In the selection criteria of materials, recycling is at the top of priorities and the industrialisation of construction is anticipated to reduce the need for human involvement, which will improve safety in this sector by its pluggable components and system configuration and sensor assisted assembly.

There are obviously a number of concerns which will need investigation, such as: the dielectricity of the material to be used for the MSTS and its electric interactions with the utilities, the design for fail-safe in the situation of leakage and diffusion of gases and liquids within the trunking. The difficulties in choosing a single material for conveying 
different utilities (as current ALM processes use single materials), the wall thickness and the R-value for thermal insulation materials between hot and cold air, together with hot and cold water interactions. All these require thorough investigation to ensure health, safety and suitability for purpose before real system can be employed in practice.

The potential risks of the proximity between services should be identified together with possible solutions. The physical integration challenge is related to the currently used manufacturing methods in construction and the design practices by architects, designers, and service engineers, materials performance, safety and longevity. Some of the materials tested within the produced prototypes showed some degrees of limitation which required more effort, time and cost to address. However, the investigation is still continuing looking potentially at the use of new materials being developed using nanotechnology engineered compositions. These will be considered for further studies and developments of more prototypes of this integrated BSs single components.

\section{Conclusion and future work}

This paper has presented the innovative concept of combining multiple services within a single trunking system for the hypothetical case of utilities distribution within a building. In a more generic form it is a multi path system that is simplified to an integrated and functional single unit. The mathematics of manifolds and Bezier surfaces has been applied to develop a 'nodes and runs' approach for routing the services and trunking through a building, whilst preserving required spacing between services. Significant complexity (geometrical and functional) underlies the routing of differing services throughout a building or any type of multi-path systems in general. The concept presented together with its manufacturability method, is capable of accommodating the principle behind the complexity. To achieve this it was first necessary to understand the elements of the complexity from their single units, and then find out how to bring them together.

The result is a proposed conceptual design for a multipath delivery systems /multiservices trunking system. Additive layer manufacturing techniques have been used to produce a laboratory prototype. Rigorous testing (as outlined above) in the laboratory is now required to fully evaluate the practical feasibility of the proposed concept, including health, safety and suitability for purpose before any such system can be applied in practice.

\section{References}


Anderson, M. J. and Whitcomb, P. J., 2000. DOE Simplified, Practical Tools for Experimentation, Productivity, Inc., Portland, Oregon. ISBN-13: 9781563272257

Bemtgen, J. M., 2006. The European Energy Approach, for Sustainable Building and cities, Unit Innovation and Technology development in Energy. Versailles, 21 November 2006, FR.

Domb, E. Report from the International TRIZ Conference, Nov, 17-18, 1998. TRIZ Journal, Dec (1998)

Hamilton, A., “Assess Design Alternatives with Axiomatic Design”. Available at: http://www.realinnovation.com/content/c080721a.asp (accessed 29 August 2012).

John, G. et al., 2002. Enhancing the Design of Building Services for Whole Life Performance. CIBSE National Technical Conference Part 2, London: Royal College of Physicians.

Liu, I.S., 1990. On Fourier's Law of Heat Conduction. Continuum Mechanics and Thermodynamics, (2), 301-305.

Liu, I.S., 1972. Method of Lagrange multipliers for exploitation of the entropy principle. Archive for Rational Mechanics and Analysis, 46, 131.

Lovell, H., 2003. Modern Methods of House Building. Parliamentary Office of Science and Technology, London.

Lovins, A.B., 1991. Profitability bating global warming. Japanese-American conference on global warming, Atlanta, June 3-4 1991.

Lovins, A.B. \& L.H., 1992. Least-cost climatic stabilisation. Annual review of energy and environment 16, 433-531.

Mann, D.L., “Axiomatic Design and TRIZ: Compatibility and Contradictions”, TRIZ Journal, June and July 1999

Noort, A., Hoek, G.F.M. and Bronsvoort, W.F. (2002), Integrating part and assembly modelling, Computer-Aided Design, 34: 899-912.

Suh, N.P.,; Ralph, E., \& Eloise, F., 2001, Axiomatic Design - Advances and Applications; ISBN13: 9780195134667 ISBN10: 0195134664; May 2001, MIT, USA.

Suh, N.P., (1990), The Principles of Design, Oxford University Press, UK, ISBN 0-19504345-6

Terninko, J., Busman, A., \& Zlotin, B., 1998, Systematic Innovation: An introduction to TRIZ., CRC Press., Florida (USA), ISBN-10: 9781574441116; 
Vaidya, P., Greden, L., Eijadi, D., McDougall, T., \& Cole, R.,, 2009. Integrated Costestimation Methodology to Support High Performance Building Design. Energy Efficiency, 2, 69-85.

The Water Supply (Water Fittings) Regulations, 1999. ISBN 011082552 7. Available at: http://www.legislation.gov.uk/uksi/1999/1506/pdfs/uksi_19991506_en.pdf (accessed 29 August 2012).

Wilson, M. J., and Martin, R. R., 2005. Mathematics of surfaces. 10th IMA international conference, Leeds, UK, 263-264. 